\title{
Architecture of an Intelligent Training System based on Virtual Environments for Electricity Distribution Substations
}

\author{
Yasmín Hernández ${ }^{1}$, Miguel Pérez Ramírez ${ }^{1}$, William Ingram Ramírez ${ }^{1}$ \\ Eric Nava Ayala ${ }^{1}$, Norma J. Ontiveros Hernández ${ }^{2}$ \\ ${ }^{1}$ Instituto Nacional de Electricidad y Energías Limpias, Tecnologías de la Información, \\ Cuernavaca, Mexico \\ ${ }^{2}$ Instituto Tecnológico de Zacatepec, Sistemas y Computación, \\ Zacatepec de Hidalgo, Mexico \\ \{mperez, myhp, jwingram\}@iie.org.mx, ericna91@gmail.com, njoh314@yahoo.com.mx
}

\begin{abstract}
In the electrical domain, qualified electricians are required since it involves danger. Training has been based on classroom courses and practice in the real installations, but it takes a long time and is costly. We propose to complement traditional training with an intelligent training system based on virtual environments in such a way that a blended training model is composed. We are looking for adaptive and innovative training; therefore we are including the characteristics and states of the student to adapt the instruction. We are developing a system to teach novice student how an electrical substation works as well as its individual compounding equipment. The proposed architecture for the intelligent training system and the development progress of the virtual environment are presented.
\end{abstract}

Keywords: Adaptive training, electrical distribution, learning environment, learning styles, virtual reality.

\section{Introduction}

Training is very important for every productive process, since if personnel are not well trained, accidents and losses could occur. In energy sector, training becomes a strategic aspect because accidents prevent the energy provision, which in turn affects industry and services sectors. Additionally, accidents could injure people. In most areas of electrical domain, the training takes a long time and is a costly process, because personnel have to move in order to practice in the actual installations. The substations are not always available because they are in operation in a regular basis. Additionally, very often there are not enough instructors available for the training demand.

We have composed a blended model with the aim of improving electrical training. The model includes the aspects of traditional training: instruction in a classroom with a certified instructor and practice in real installations; and the novel component is conformed by intelligent training systems with virtual environments which allow 
learning the electricity topics and practicing the maneuvers before practicing in real world. The systems provide virtual representations of the electrical installations. To date, we have developed several virtual reality systems for learning maneuvers in domains such as electrical tests in substations [1] and energized lines [2].

Now, we are developing a system to teach about the individual equipment composing a distribution substation, in order to teach novice electricians how they work and how they contribute to the whole process of the substation. The system presents the lessons with textual and auditory explanations in a virtual environment, and we are including elements of augmented reality.

There are diverse advantages of the virtual reality, one of them strives in that it allows to visit places which are difficult to get or physically impossible to visit. On the other hand, the augmented reality, allows having information which are not explicitly present in the real environment. We are trying to integrate these features in a low-cost training system.

In this paper, we describe the proposed architecture for the intelligent training system and the work in progress in the virtual environment development. The rest of the paper is organized as follows: Section 2 describes the architecture of the proposed model. Section 3 describes the virtual environment. Section 4 includes a discussion. Finally, section 5 presents some conclusions.

\section{Related Work}

There are some works relating Virtual Reality and Substations. For instance in [3] is reported a work in progress; relevant here is that the authors consider that immersion is mandatory which might be inaccurate since besides visual and auditory feedback, the other senses of users are poorly stimulated, smell and taste are still under research. Full immersion would provide feedback to all five senses, in such a way that users would have the experiencing of being inside of a synthetic environment.

Furthermore the cost of immersion might still be prohibitive for companies with thousands of users, although immersion might still be attractive for demonstration purposes or gamming. Some advantages of VR already mentioned here are included in [4], within a substation in a 3D virtual environment. In [5] a Chinese company mentions other advantages of applying VR to train substation operators such as improving substation operation, provide brake operation ability, strengthen the safety consciousness, and develop strict compliance work style. A closer functionality to our system is reported in [6], where is mentioned that each component of the substation is reproduced in the simulation model, including the behavior laws associated with it, so the complete functionality of the sub-station can be simulated.

\section{$3 \quad$ Electrical Distribution System}

The electrical distribution substations receive high tension of $115 \mathrm{kV}$ and delivers medium tension of $24 \mathrm{kV}$. The substation reduces the voltage which then is transported by the medium tension distribution poles to the cities for industrial and domestic use. Here the $24 \mathrm{kV}$ are reduced to $127 \mathrm{v}$, which is the Mexican standard, by the small 
transformers located on the distribution poles. In an overall view, Fig. 1 depicts the main operation of an electrical substation.

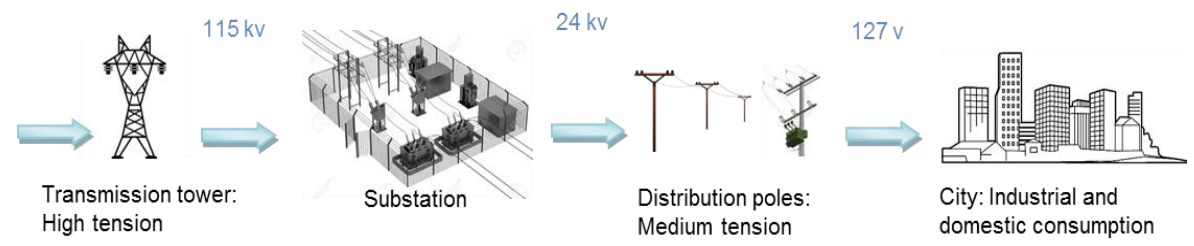

Fig. 1. Operation of the electrical distribution system. The substations receive high tension of $115 \mathrm{Kv}$ and delivers medium tension of $24 \mathrm{Kv}$. The voltage is transported by the medium tension distribution poles to the cities for the industrial and domestic use. The $24 \mathrm{Kv}$ are reduced to $127 \mathrm{v}$, by the small transformers located on the distribution poles. Partial images taken from [7].

\section{$4 \quad$ Intelligent Training System Architecture}

The traditional training has been successful; however it takes a long time and therefore it is costly. We are composing a blended model to support traditional training. As can be observed in Fig. 2, in this new model we include intelligent learning systems to provide an adaptive training which in turn minimizes costs and hours of training; also we include virtual environments to provide trainees with a realistic scenario to practice the electrical maneuvers. As we mention, we have developed several systems for different areas of electricity domain, here we are presenting a system to teach how an electricity distribution substation works, its components and relationships. More details about the model can be found in [8].

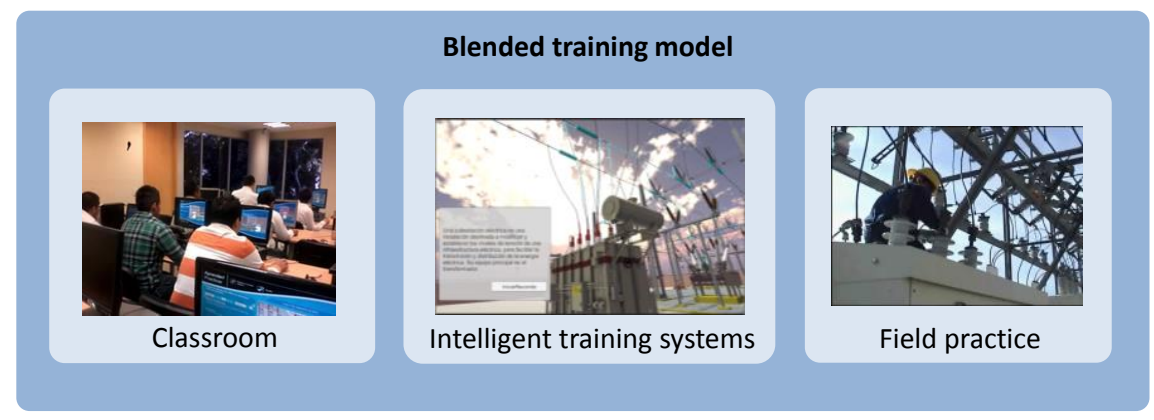

Fig. 2. Blended training model. The model is composed by a) instruction in a classroom with a certified instructor, b) practice in real installations; and c) intelligent training systems with virtual environments.

The new component, the intelligent training systems, complements the classroom classes. The trainees receive the lessons by an instructor and they can practice the maneuvers with the system. The system allows distance training since electricians can learn by themselves about the operation of a substation even before they are enrolled in 
a course or have access to a real substation. The architecture of the system is presented in Fig. 3.

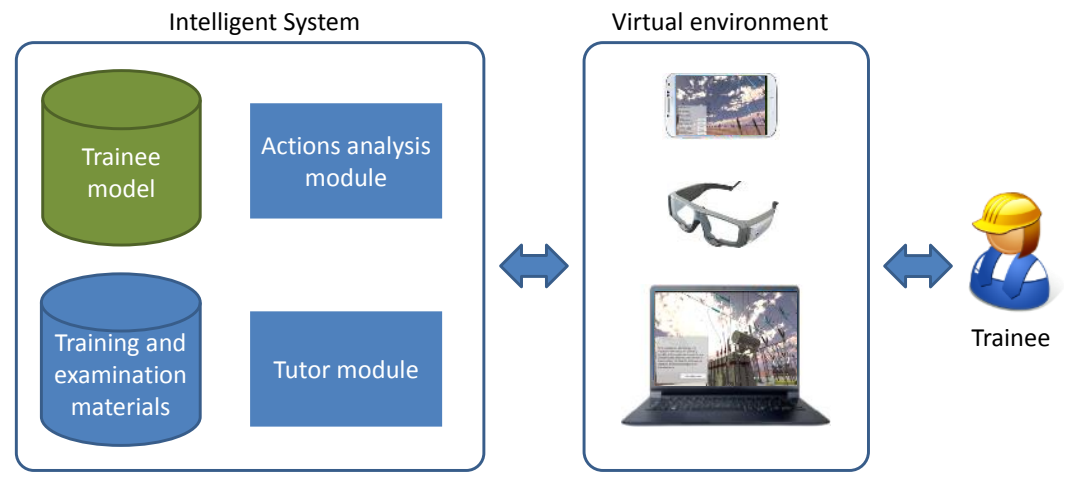

Fig. 3. Architecture of the intelligent training system for electricity distribution substations. The system is composed by two principal components: the intelligent system and the virtual environment. The first one includes two modules: the analysis of actions of the trainee and the tutor module. The virtual environment is a virtual representation of a real substation, and it stands for the interface with the trainee.

The intelligent training system provides adaptation and personalized instruction based on the state and characteristics of the trainees. To do that, the system maintains the trainee model, which is a representation of the trainee; it contains the characteristics of the trainee and what he knows about the subject matter. For the time being, we are including the learning styles [9] of the trainees to present the explanations about the equipment.

A repository stores the training and examination materials. The materials are designed in different degrees of detail; and in different formats to accomplish different learning styles. The training and examining material were developed and designed by a team of experts.

The analysis of actions module determines the equipment which is the focus of the interest of the trainee base on clicks and gazes. This module relies on artificial intelligence algorithms to establish the equipment which is the interest for the trainee. Technologies like eye tracking and augmented reality are being analyzed for detecting the point of interest of the trainee.

The tutor model receives the knowledge about the interest of the trainee and establishes what to teach according to the knowledge about the trainee, stored in the trainee model.

\subsection{Trainee Model}

We have developed a student model that considers the affect and knowledge to decide the next tutorial action. The tutor monitors the affective state of students and reacts in consequence. Now, we want to have more adaptive instruction identifying the learning style of students and to provide students with an instruction according with their learning style [10]. 
We based our approach on Felder-Silverman learning styles model [11, 12, 13] which proposes the following learning styles: active, reflective, sensing, intuitive, visual, verbal, sequential, and global. The model also proposes teaching rules for each learning style [9].

In this way, the learning style determines the type of explanations to be presented to the student. However the affect, knowledge and interest determine what should be explained to the trainee.

In this way, our integration approach allows building intelligent tutors that are adaptive in response to the knowledge state, the affective state and the learning style of the students.

\section{$5 \quad$ Virtual Environment}

Virtual reality provides advantageous features which make it a good tool to create learning environments. Among other features, we can mention, it allows active learning, provides simultaneous stimulus to different leaning channel for different kinds of students namely visual, auditory and kinesthetic.

The virtual environment allows free navigation throughout the substation, and trainees can first follow the correct sequence guided by the system and then go to particular components in order to review how specific equipment work. Free navigation makes also possible the inspection of the substation.

The system provides a comprehensive view of the operation of a substation. This includes detailed information about each component through the substation. This is achieved by providing visual, text and audio information and enhanced by $3 \mathrm{D}$ animations.

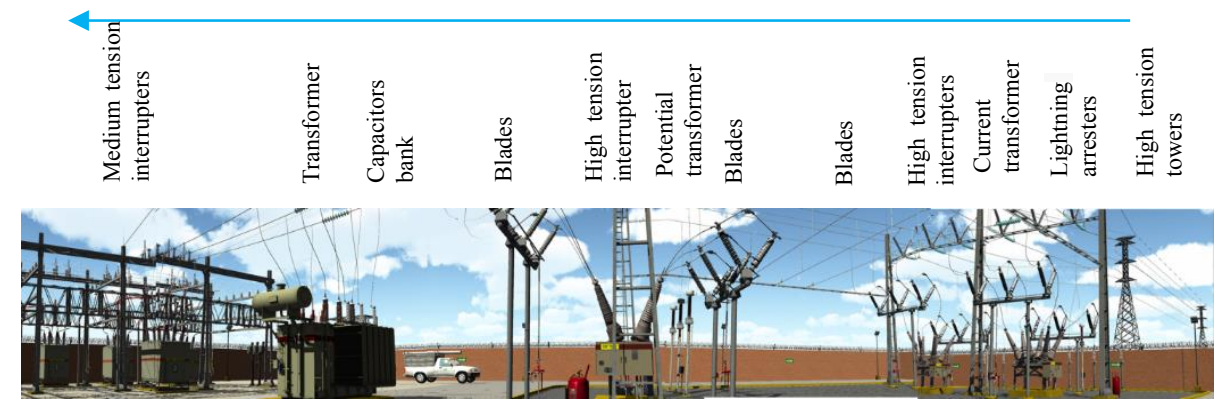

Fig. 4. Virtual representation of an electrical distribution substation. The electricity is delivered to substation by the high tension tower, and it run through the primary equipment.

Following the energy flow shown in Fig. 4, the operation of a substation is accounted for a sequence starting in the high tension towers, then the lightning arresters, current transformers, high tension interrupters (breakers), potential transformers, transformer, capacitors bank, medium tension breakers, and so on.

For instance, the system provides information about lighting arresters (LA) which are the first primary equipment in the substation which receives the high tension potential. The written information explain how the LA work, then some animations are 
triggered so that first: a particles animation illustrates the path of the current through the cables until it reaches the LA, and second: under demand by using a button the user can trigger another animation where the LA turn transparent so that the user can see how the internal devices work. Here it is explained that when the LA receive a tension overload, the LA close the circuit to guide the voltage overload towards the ground (Fig. 5).

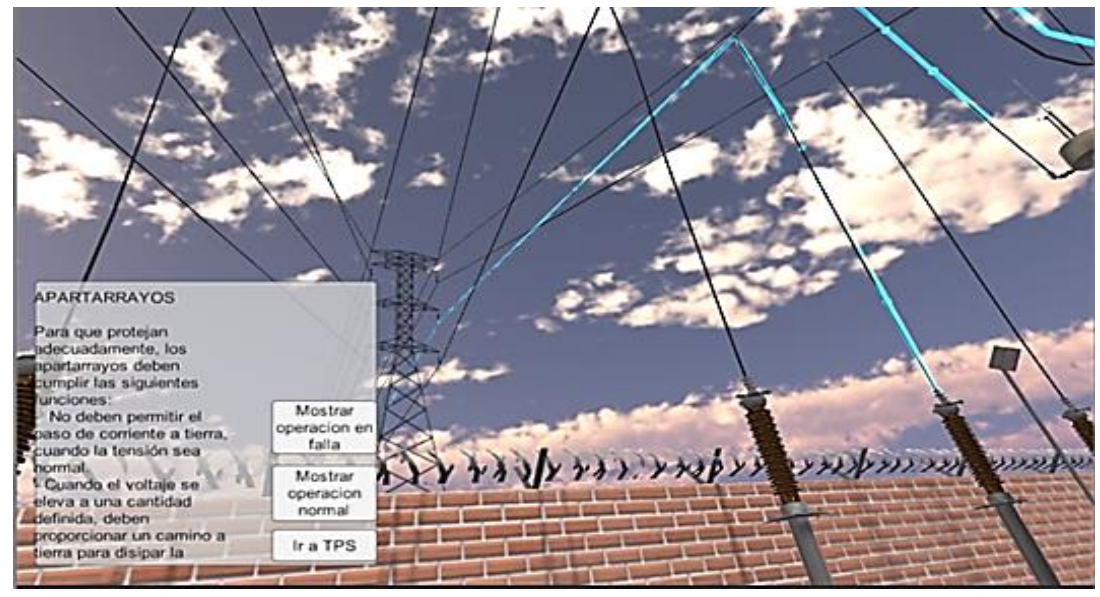

Fig. 5. The system provides information about lighting arrester. A particles animation illustrates the path of the tension. Thus, the system shows how the lighting arresters work in case of over tension based on the animation of the running tension.

As another example that deserves to be mentioned here, has to do with the transformer, since this is the core of a substation. Again using animations illustrating the path of the current, the system shows how on one side it receives the high tension and on the other side it delivers medium tension. In the explanation the transformer turns transparent so that the user will be able to see the inside of it, and an animation will illustrate how the tension is transformed (Fig. 6).

Contingency situations (CS) occur when something goes wrong in the substation, when primary equipment fails due to different possible causes, for instance lightings, voltage overload, short circuit originated by fauna, and so on. CS are associated to corrective maintenance, that is to say some equipment fails and it needs to be fixed or replaced.

Our system also is planned to include training to perform operations in CS within a collaborative environment, where we can have both the console of the instructor and the console of the student. Thus the instructor would be able to introduce specific CS and observe how students proceed to fix the situation. This is suitable for the implementations not only of active learning but naturally for the implementation of problem-based learning.

As an example of a contingency situation we can mention a tripped breaker. In case the breaker is not under observation, here a student must verify whether or not the breaker was operated by a protection (R50). If so, he must restore the relay flags and make a revision of the equipment informing to the operator. In case there is something 
wrong in the substation, he should start the maneuvers to bypass the equipment and isolate the failure. Then after correcting the problem, the equipment must be restored into the circuit [14].

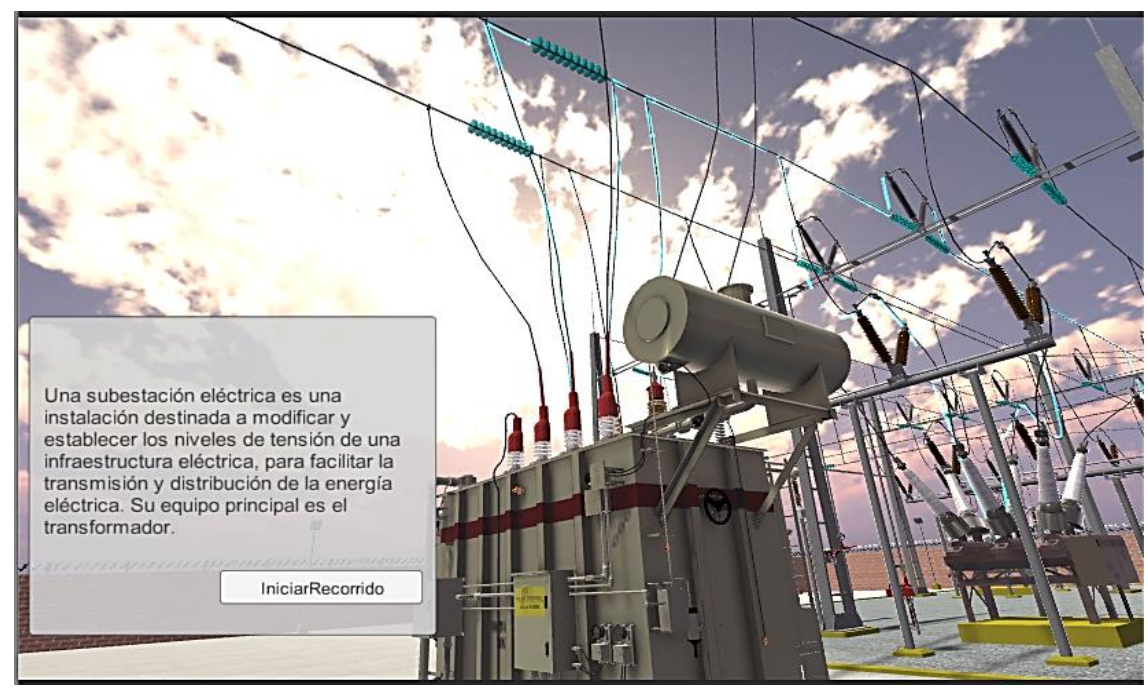

Fig. 6. The system shows how a transformer works. The transformer is the core of the substation since it reduces the high tension to medium tension. The system will show how the transformer works by using animations and transparencies to the see the inner parts of the transformer.

\section{Conclusions and Future Work}

Thus far, we can navigate or inspect the substation. We have different animations to show closing and opening blades and particles to illustrate the current flow. The system is also able to show textual and auditory information to explain how primary equipment works in the substation. In parallel in a different application we have implemented the collaborative learning environment which we will include in this system. Finally we have developed the controls of an animated agent representing an electrician. Thus, a trainee can move the electrician and control it like in a game. This entire infrastructure will be used to add the collaborative learning environment and the capability to train contingency situations. The system is intended to provide novice students a full view of the operation of a substation and some contingence situations.

There are at least three main functionalities that we will integrate to the system, namely: collaboration, animated agents, augmented reality.

In another development we have successfully implemented the simulation environment including the instructor and student consoles by using the multiplayer potential of Unity (https://unity3d.com/es), the game engine that we are using. We want to replicate and integrate also this functionality into this system. We have also worked on an animated agent that we pretend to integrate to the system as an animated agent.

We pretend shortly to use augmented reality to enhance performance of electricians. Once we have an electrical substation and different animations regarding the electrical 
tests, we want to include the image processing to identify different primary equipment in the substation and together with the test sequence we can organize the information and then by using some glasses or a Smartphone to deliver 3D images and explanation so that the electricians are helped to perform maintenance procedures and electrical tests. In fact this would lead us to a different application.

The system is being developed using 3DSMax and Blender for 3D modeling and Unity as an integrator.

To incorporate learning styles in the adaptation of tutoring systems allows identifying the best tutorial action given the students' preferences, strategies, experience, and so on.

\section{References}

1. Pérez, M., Gallegos, S., Zayas, B., Islas, E., Hernández, I., Espinoza, C.: Capacitación en pruebas a Subestaciones de Distribución utilizando Realidad Virtual. In: Proc. of XI CIINDET 2014, Cuernavaca, Mexico (2014)

2. Ayala, A., Galván, I., Arroyo, G., Pérez, M., Muñoz, J.: Virtual reality training system for maintenance and operation of high-voltage overhead power lines. Virtual Reality, 20(1), pp. 27-40 (2016)

3. Tanaka, E., Paludo, J., Cordeiro, C., Domingues, L., Gadbem, E., Euflausino, A.: Using immersive virtual reality for electrical substation training. In: Proc. of International Conference e-Learning 2015, Las Palmas de Gran Canaria, Spain (2015)

4. Sun, F. J., Chen, H., Liu, H. J: Research of Visualized 3d Substation Simulation Based on Virtual Reality Technology. Applied Mechanics and Materials, pp. 568-570, 1834-1838 (2014)

5. Shandong: The virtual reality and training system of substation. http://runqingtech.com/enweb/ywbdzxnxsfzpxxt.htm Accessed in August 2016 (2016)

6. Romero, G., Maroto, J., Felez, J., Cabanellas, J. M., Martinez, M. L., Carretero, A.: Virtual reality applied to a full simulator of electrical sub-stations. Electric Power System Research, 78(3), pp. 409-417 (2008)

7. Subgerencia Redes de Distribución: Planos del montaje transformador trifásico en poste. https://www.epm.com.co/site/Portals/0/Users/037/93/293/RA2-026.pdf, Accessed on August 2016 (2013)

8. Hernández, Y., Pérez, M.: Open Student Model for Blended Training in the Electrical Tests Domain. In: Proc. of MICAI 2015, LNAI 9414, pp. 195-207 (2015)

9. Hernández, Y., Rodríguez, G.: Learning Styles Theory for Intelligent Learning Environments. In: Proc. of CSEDU 2011, 1, pp. 456-459 (2011)

10. Hernández, Y., Arroyo-Figueroa, G., Sucar, L. E.: A Model of Affect and Learning for Intelligent Tutors. Journal of Universal Computer Science, 21(7), pp. 912-934 (2015)

11. Blouin, T.: Felder-Silverman Learning Styles Model. http://chat.carleton.ca/ tblouin/ Felder/felder.html, Accessed on August 2016 (2010)

12. Felder, R. M, Silverman, L. K.: Learning and teaching styles in engineering education. Eng. Educ., 78(7), pp. 674-681(1988)

13. Felder, R. M., Soloman, B. A.: Learning styles and strategies. NCSU (1993)

14. Martínez, Mendoza, Gonzalez, Riveros, Romero, Smith, Alfonsin, Luna, Barrero, Carmona, Martínez, Domit, Pérez, Rasso, Lopez, Serrano, Medecigo, Lee W.: Instructivo de operación para alimentadores aéreos en disturbio (2004) 